\title{
A Refined Method for Estimating the Global Hölder Exponent
}

T. Kleyntssens, D. Kreit \& S. Nicolay

ITNG 2016

Las Vegas, April 11-13, 2016 
A function $f \in L^{\infty}\left(\mathbb{R}^{d}\right)$ belongs to the Hölder space $\Lambda^{s}\left(\mathbb{R}^{d}\right)$ iff there exists a constant $C$ such that for each $x \in \mathbb{R}^{d}$, there exists a polynomial $P_{x}$ of degree at most $s$ for which

$$
\left|f(x+h)-P_{x}(h)\right| \leq C|h|^{s} .
$$


A function $f \in L^{\infty}\left(\mathbb{R}^{d}\right)$ belongs to the Hölder space $\Lambda^{s}\left(\mathbb{R}^{d}\right)$ iff there exists a constant $C$ such that for each $x \in \mathbb{R}^{d}$, there exists a polynomial $P_{x}$ of degree at most $s$ for which

$$
\left|f(x+h)-P_{x}(h)\right| \leq C|h|^{s} .
$$

Since these spaces are embedded, on can define the Hölder exponent of $f$ as follows :

$$
H_{f}=\sup \left\{s: f \in \Lambda^{s}\left(\mathbb{R}^{d}\right)\right\} .
$$


Example: a sample path of the Brownian motion has a Hölder exponent equal to $1 / 2$ a.s.

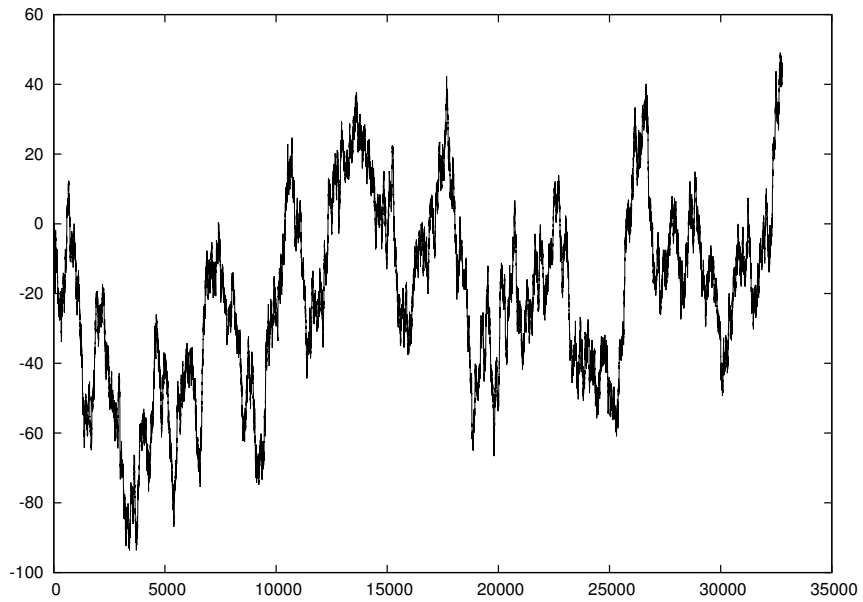


Example: a sample path of the fractional Brownian motion with Hurst index 0.3 has a Hölder exponent equal to 0.3 a.s.

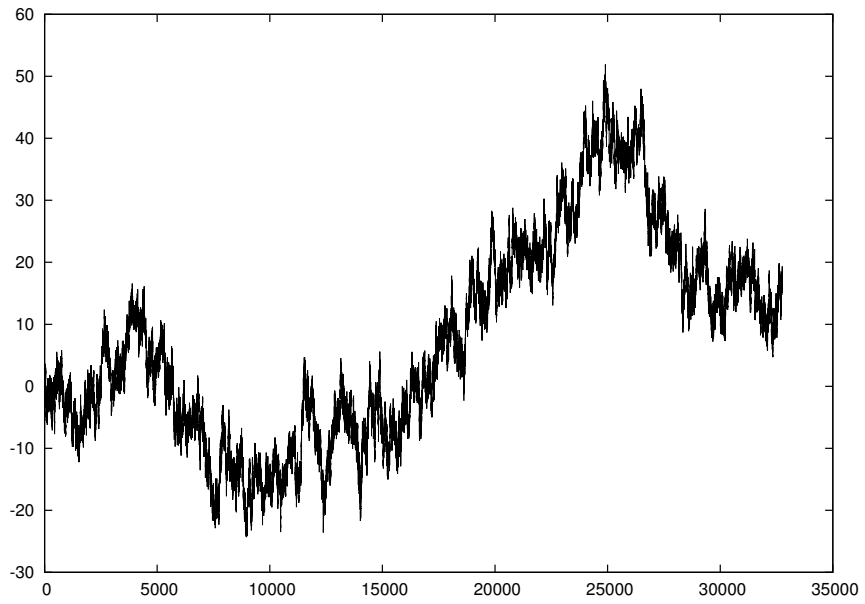


Example: a sample path of the fractional Brownian motion with Hurst index 0.7 has a Hölder exponent equal to 0.7 a.s.

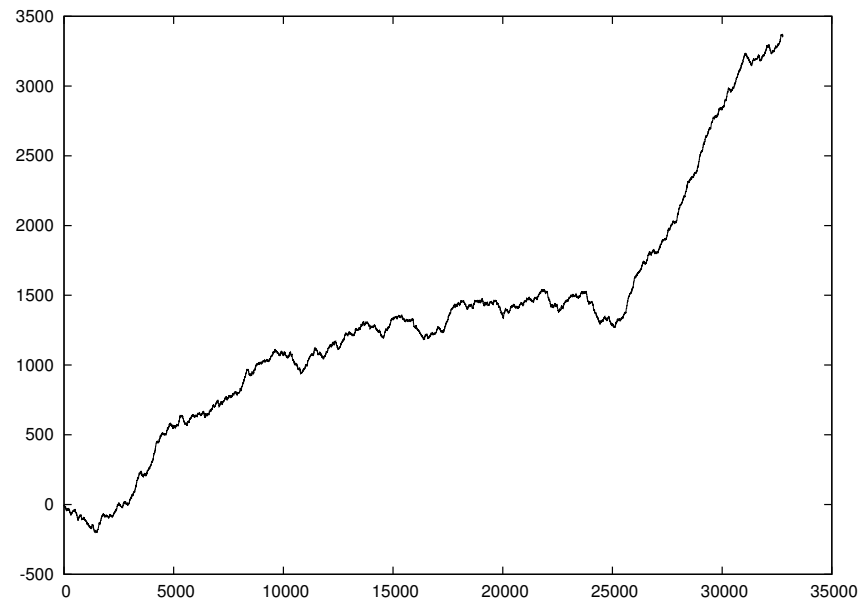


The regularity increases with the Hölder exponent
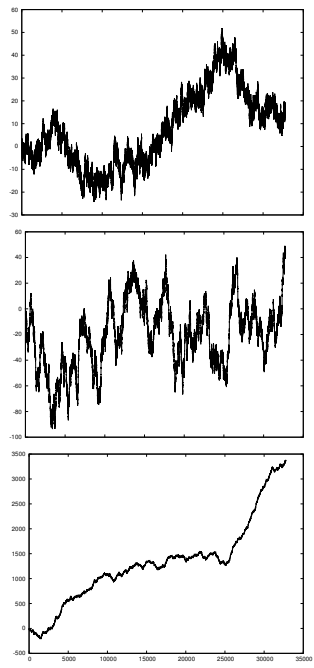
A natural generalization consists in replacing the exponent $s$ with a sequence $\sigma$ satisfying some properties

$$
B_{p, q}^{s}\left(\mathbb{R}^{d}\right) \longrightarrow \text { generalization } \longrightarrow B_{p, q}^{1 / \sigma}\left(\mathbb{R}^{d}\right)
$$


A natural generalization consists in replacing the exponent $s$ with a sequence $\sigma$ satisfying some properties

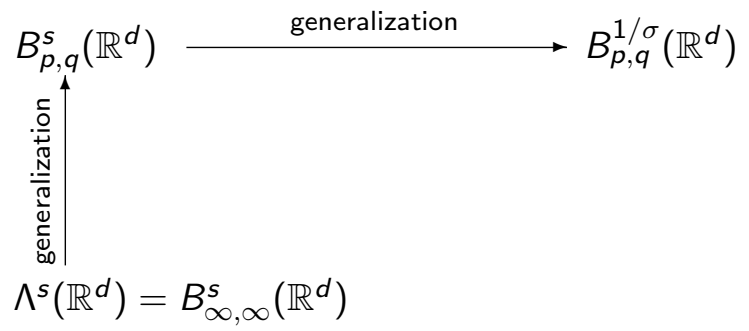


A natural generalization consists in replacing the exponent $s$ with a sequence $\sigma$ satisfying some properties

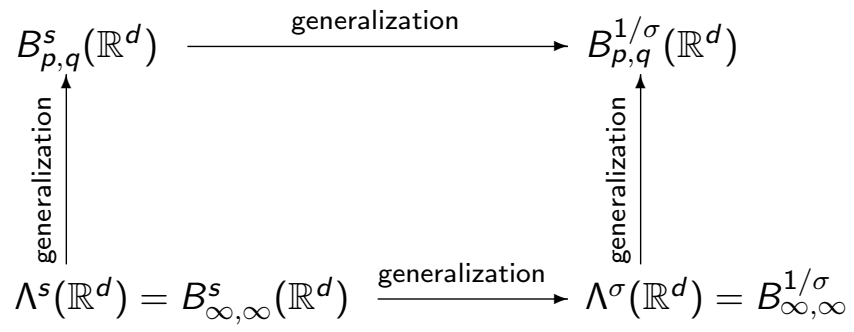


Such a generalization could help to better characterize some specific functions or processes 
Such a generalization could help to better characterize some specific functions or processes

For example, the sample path of a Brownian motion $W$ satisfies

$$
|W(t+h)-W(t)| \leq C \sqrt{|h| \log |\log | h||}
$$

for some constant $C>\sqrt{2}$ a.s. 
Such a generalization could help to better characterize some specific functions or processes

For example, the sample path of a Brownian motion $W$ satisfies

$$
|W(t+h)-W(t)| \leq C \sqrt{|h| \log |\log | h||}
$$

for some constant $C>\sqrt{2}$ a.s.

Therefore, this method could be used to detect if a process is issued from a Brownian motion 
A sequence of real positive numbers is called admissible if

$$
\frac{\sigma_{j+1}}{\sigma_{j}}
$$

is bounded. 
A sequence of real positive numbers is called admissible if

$$
\frac{\sigma_{j+1}}{\sigma_{j}}
$$

is bounded.

For such a sequence, we set

$$
\underline{s}(\sigma)=\lim _{j} \frac{\log _{2}\left(\inf _{k \in \mathbb{N}} \frac{\sigma_{j+k}}{\sigma_{j}}\right)}{j}
$$

and

$$
\bar{s}(\sigma)=\lim _{j} \frac{\log _{2}\left(\sup _{k \in \mathbb{N}} \frac{\sigma_{j+k}}{\sigma_{j}}\right)}{j} .
$$


- the open unit ball centered at the origin is denoted $B$,

- the set of polynomials of degree at most $n$ is denoted $\mathbf{P}[n]$,

- $[s]=\sup \{n \in \mathbb{Z}: n \leq s\}$, 
- the open unit ball centered at the origin is denoted $B$,

- the set of polynomials of degree at most $n$ is denoted $\mathbf{P}[n]$,

- $[s]=\sup \{n \in \mathbb{Z}: n \leq s\}$,

- if $f$ is defined on $\mathbb{R}^{d}$,

$$
\Delta_{h}^{1} f(x)=f(x+h)-f(x)
$$


- the open unit ball centered at the origin is denoted $B$,

- the set of polynomials of degree at most $n$ is denoted $\mathbf{P}[n]$,

- $[s]=\sup \{n \in \mathbb{Z}: n \leq s\}$,

- if $f$ is defined on $\mathbb{R}^{d}$,

$$
\Delta_{h}^{1} f(x)=f(x+h)-f(x)
$$

and

$$
\Delta_{h}^{n+1} f(x)=\Delta_{h}^{1} \Delta_{h}^{n} f(x),
$$

for any $x, h \in \mathbb{R}^{d}$ 


\section{Definition}

Let $s>0$ and $\sigma$ be an admissible sequence; a function $f \in L^{\infty}\left(\mathbb{R}^{d}\right)$ belongs to $\Lambda^{\sigma, M}\left(\mathbb{R}^{d}\right)$ iff there exists $C>0$ s.t.

$$
\sup _{|h| \leq 2^{-j}}\left\|\Delta_{h}^{[M]+1} f\right\|_{\infty} \leq C \sigma_{j}
$$




\section{Definition}

Let $s>0$ and $\sigma$ be an admissible sequence; a function $f \in L^{\infty}\left(\mathbb{R}^{d}\right)$ belongs to $\Lambda^{\sigma, M}\left(\mathbb{R}^{d}\right)$ iff there exists $C>0$ s.t.

$$
\sup _{|h| \leq 2^{-j}}\left\|\Delta_{h}^{[M]+1} f\right\|_{\infty} \leq C \sigma_{j}
$$

\section{Proposition}

Let $s>0$ and $\sigma$ be an admissible sequence; a function $f \in L^{\infty}\left(\mathbb{R}^{d}\right)$ belongs to $\Lambda^{\sigma, M}\left(\mathbb{R}^{d}\right)$ iff there exists $C>0$ s.t.

$$
\inf _{P \in \mathbf{P}_{[M]}}\|f-P\|_{L^{\infty}\left(2^{-j} B+x\right)} \leq C \sigma_{j}
$$

for any $x \in \mathbb{R}^{d}$ and any $j \in \mathbb{N}$. 
- One sets $\Lambda^{\sigma}\left(\mathbb{R}^{d}\right)=\Lambda^{\sigma, \bar{s}\left(\sigma^{-1}\right)}\left(\mathbb{R}^{d}\right)$ 
- One sets $\Lambda^{\sigma}\left(\mathbb{R}^{d}\right)=\Lambda^{\sigma, \bar{s}\left(\sigma^{-1}\right)}\left(\mathbb{R}^{d}\right)$

- For $s>0$, one has $\Lambda^{s}\left(\mathbb{R}^{d}\right)=\Lambda^{\sigma}\left(\mathbb{R}^{d}\right)$ with $\sigma_{j}=2^{-j s}$ 
- One sets $\Lambda^{\sigma}\left(\mathbb{R}^{d}\right)=\Lambda^{\sigma, \bar{s}\left(\sigma^{-1}\right)}\left(\mathbb{R}^{d}\right)$

- For $s>0$, one has $\Lambda^{s}\left(\mathbb{R}^{d}\right)=\Lambda^{\sigma}\left(\mathbb{R}^{d}\right)$ with $\sigma_{j}=2^{-j s}$

- The application

$$
|f|_{\Lambda^{\sigma, M}}=\sup _{j}\left(\sigma_{j}^{-1} \sup _{|h| \leq 2^{-j}}\left\|\Delta_{h}^{[M]+1} f\right\|_{L^{\infty}}\right)
$$

defines a semi-norm on $\Lambda^{\sigma, M}$ 
- One sets $\Lambda^{\sigma}\left(\mathbb{R}^{d}\right)=\Lambda^{\sigma, \bar{s}\left(\sigma^{-1}\right)}\left(\mathbb{R}^{d}\right)$

- For $s>0$, one has $\Lambda^{s}\left(\mathbb{R}^{d}\right)=\Lambda^{\sigma}\left(\mathbb{R}^{d}\right)$ with $\sigma_{j}=2^{-j s}$

- The application

$$
|f|_{\Lambda^{\sigma, M}}=\sup _{j}\left(\sigma_{j}^{-1} \sup _{|h| \leq 2^{-j}}\left\|\Delta_{h}^{[M]+1} f\right\|_{L^{\infty}}\right)
$$

defines a semi-norm on $\Lambda^{\sigma, M}$

Therefore $\|f\|_{\Lambda^{\sigma, M}}=\|f\|_{L^{\infty}}+|f|_{\Lambda^{\sigma, M}}$ is a norm on this space 
- One sets $\Lambda^{\sigma}\left(\mathbb{R}^{d}\right)=\Lambda^{\sigma, \bar{s}\left(\sigma^{-1}\right)}\left(\mathbb{R}^{d}\right)$

- For $s>0$, one has $\Lambda^{s}\left(\mathbb{R}^{d}\right)=\Lambda^{\sigma}\left(\mathbb{R}^{d}\right)$ with $\sigma_{j}=2^{-j s}$

- The application

$$
|f|_{\Lambda^{\sigma, M}}=\sup _{j}\left(\sigma_{j}^{-1} \sup _{|h| \leq 2^{-j}}\left\|\Delta_{h}^{[M]+1} f\right\|_{L^{\infty}}\right)
$$

defines a semi-norm on $\Lambda^{\sigma, M}$

Therefore $\|f\|_{\Lambda^{\sigma, M}}=\|f\|_{L^{\infty}}+|f|_{\Lambda^{\sigma, M}}$ is a norm on this space

\section{Theorem}

Let $M>0$; the space $\left(\Lambda^{\sigma, M}\left(\mathbb{R}^{d}\right),\|\cdot\|_{\Lambda^{\sigma, M}}\right)$ is a Banach space 
- One sets $\Lambda^{\sigma}\left(\mathbb{R}^{d}\right)=\Lambda^{\sigma, \bar{s}\left(\sigma^{-1}\right)}\left(\mathbb{R}^{d}\right)$

- For $s>0$, one has $\Lambda^{s}\left(\mathbb{R}^{d}\right)=\Lambda^{\sigma}\left(\mathbb{R}^{d}\right)$ with $\sigma_{j}=2^{-j s}$

- The application

$$
|f|_{\Lambda^{\sigma, M}}=\sup _{j}\left(\sigma_{j}^{-1} \sup _{|h| \leq 2^{-j}}\left\|\Delta_{h}^{[M]+1} f\right\|_{L^{\infty}}\right)
$$

defines a semi-norm on $\Lambda^{\sigma, M}$

Therefore $\|f\|_{\Lambda^{\sigma, M}}=\|f\|_{L^{\infty}}+|f|_{\Lambda^{\sigma, M}}$ is a norm on this space

\section{Theorem}

Let $M>0$; the space $\left(\Lambda^{\sigma, M}\left(\mathbb{R}^{d}\right),\|\cdot\|_{\Lambda^{\sigma, M}}\right)$ is a Banach space

For example, a sample path of the Brownian motion belongs to $\Lambda^{\sigma}(\mathbb{R})$ with $\sigma=\left(2^{-j / 2} \sqrt{\log j}\right)_{j}$ a.s. 


\section{Theorem}

Let $\sigma$ be an admissible sequence and $M, N$ be two positive integers such that

$$
N<\underline{s}\left(\sigma^{-1}\right) \leq \bar{s}\left(\sigma^{-1}\right)<M ;
$$

Any element of $\Lambda^{\sigma}\left(\mathbb{R}^{d}\right)$ is equal a.e. to a function $f \in C^{N}\left(\mathbb{R}^{d}\right)$ satisfying $D^{\alpha} f \in L^{\infty}\left(\mathbb{R}^{d}\right)$ for any multi-index $\alpha$ such that $|\alpha| \leq N$ and

$$
\sup _{|h| \leq 2^{-j}}\left\|\Delta_{h}^{M-|\alpha|} D^{\alpha} f\right\|_{L^{\infty}} \leq C 2^{j|\alpha|} \sigma_{j}
$$

for any $j \in \mathbb{N}$ and $|\alpha| \leq N$. 


\section{Theorem}

Let $\sigma$ be an admissible sequence and $M, N$ be two positive integers such that

$$
N<\underline{s}\left(\sigma^{-1}\right) \leq \bar{s}\left(\sigma^{-1}\right)<M ;
$$

Any element of $\Lambda^{\sigma}\left(\mathbb{R}^{d}\right)$ is equal a.e. to a function $f \in C^{N}\left(\mathbb{R}^{d}\right)$ satisfying $D^{\alpha} f \in L^{\infty}\left(\mathbb{R}^{d}\right)$ for any multi-index $\alpha$ such that $|\alpha| \leq N$ and

$$
\sup _{|h| \leq 2^{-j}}\left\|\Delta_{h}^{M-|\alpha|} D^{\alpha} f\right\|_{L^{\infty}} \leq C 2^{j|\alpha|} \sigma_{j}
$$

for any $j \in \mathbb{N}$ and $|\alpha| \leq N$.

Conversely, if $f \in L^{\infty}\left(\mathbb{R}^{d}\right) \cap C^{N}\left(\mathbb{R}^{d}\right)$ satisfies the previous inequality for $|\alpha|=N$ then $f$ belongs to $\Lambda^{\sigma}\left(\mathbb{R}^{d}\right)$. 
Under some general conditions, there exist a function $\phi$ and $2^{d}-1$ functions $\psi^{(i)}$ called wavelets s.t.

$$
\left\{\phi(\cdot-k): k \in \mathbb{Z}^{d}\right\} \bigcup\left\{\psi^{(i)}\left(2^{j} \cdot-k\right): k \in \mathbb{Z}^{d}, j \in \mathbb{N}_{0}\right\}
$$

forms an orthogonal basis of $L^{2}\left(\mathbb{R}^{d}\right)$. 
Under some general conditions, there exist a function $\phi$ and $2^{d}-1$ functions $\psi^{(i)}$ called wavelets s.t.

$$
\left\{\phi(\cdot-k): k \in \mathbb{Z}^{d}\right\} \bigcup\left\{\psi^{(i)}\left(2^{j} \cdot-k\right): k \in \mathbb{Z}^{d}, j \in \mathbb{N}_{0}\right\}
$$

forms an orthogonal basis of $L^{2}\left(\mathbb{R}^{d}\right)$. Any function $f \in L^{2}\left(\mathbb{R}^{d}\right)$ can be decomposed as follows,

$$
f(x)=\sum_{k \in \mathbb{Z}^{d}} c_{k} \phi(x-k)+\sum_{j \geq 0, k \in \mathbb{Z}^{d}, 1 \leq i<2^{d}} c_{j, k}^{(i)} \psi^{(i)}\left(2^{j} x-k\right),
$$

with

$$
C_{k}=\int f(x) \phi(x-k) d x, \quad c_{j, k}^{(i)}=2^{d j} \int f(x) \psi^{(i)}\left(2^{j} x-k\right) d x .
$$


Under some general conditions, there exist a function $\phi$ and $2^{d}-1$ functions $\psi^{(i)}$ called wavelets s.t.

$$
\left\{\phi(\cdot-k): k \in \mathbb{Z}^{d}\right\} \bigcup\left\{\psi^{(i)}\left(2^{j} \cdot-k\right): k \in \mathbb{Z}^{d}, j \in \mathbb{N}_{0}\right\}
$$

forms an orthogonal basis of $L^{2}\left(\mathbb{R}^{d}\right)$. Any function $f \in L^{2}\left(\mathbb{R}^{d}\right)$ can be decomposed as follows,

$$
f(x)=\sum_{k \in \mathbb{Z}^{d}} c_{k} \phi(x-k)+\sum_{j \geq 0, k \in \mathbb{Z}^{d}, 1 \leq i<2^{d}} c_{j, k}^{(i)} \psi^{(i)}\left(2^{j} x-k\right),
$$

with

$$
C_{k}=\int f(x) \phi(x-k) d x, \quad c_{j, k}^{(i)}=2^{d j} \int f(x) \psi^{(i)}\left(2^{j} x-k\right) d x .
$$

In what follows, we will assume that the wavelets are the Daubechies wavelets 


\section{Theorem}

Let $\sigma$ be an admissible sequence such that $\underline{s}\left(\sigma^{-1}\right)>0$. If $f$ belongs to $\Lambda^{\sigma}\left(\mathbb{R}^{d}\right)$, there exists a constant $C>0$ such that

$$
\left|C_{k}\right|<C \quad \text { and } \quad\left|c_{j, k}^{(i)}\right| \leq C \sigma_{j}
$$

for any $j \in \mathbb{N}$ any $k \in \mathbb{Z}^{d}$ and any $i \in\left\{1, \ldots, 2^{d-1}\right\}$. 


\section{Theorem}

Let $\sigma$ be an admissible sequence such that $\underline{s}\left(\sigma^{-1}\right)>0$. If $f$ belongs to $\Lambda^{\sigma}\left(\mathbb{R}^{d}\right)$, there exists a constant $C>0$ such that

$$
\left|C_{k}\right|<C \quad \text { and } \quad\left|c_{j, k}^{(i)}\right| \leq C \sigma_{j}
$$

for any $j \in \mathbb{N}$ any $k \in \mathbb{Z}^{d}$ and any $i \in\left\{1, \ldots, 2^{d-1}\right\}$.

Conversely, if $f \in L_{\mathrm{loc}}^{\infty}\left(\mathbb{R}^{d}\right)$ and if the previous relations hold, then $f$ belongs to $\Lambda^{\sigma}\left(\mathbb{R}^{d}\right)$. 
Let $\Psi_{j}$ denote the set of wavelet coefficients at scale $j$. The power spectrum of $f$ is defined as follows

$$
S_{f}(j)=\sqrt{\frac{1}{\# \Psi_{j}} \sum_{i, k}\left|c_{j, k}^{(i)}\right|^{2}}
$$


Let $\Psi_{j}$ denote the set of wavelet coefficients at scale $j$. The power spectrum of $f$ is defined as follows

$$
S_{f}(j)=\sqrt{\frac{1}{\# \Psi_{j}} \sum_{i, k}\left|c_{j, k}^{(i)}\right|^{2}}
$$

If $f$ is associated to a Hölder exponent to $H_{f}=h$, one should have

$$
S_{f}(j) \sim C 2^{-j h}
$$

for some constant $C$ 
Let $\Psi_{j}$ denote the set of wavelet coefficients at scale $j$. The power spectrum of $f$ is defined as follows

$$
S_{f}(j)=\sqrt{\frac{1}{\# \Psi_{j}} \sum_{i, k}\left|c_{j, k}^{(i)}\right|^{2}}
$$

If $f$ is associated to a Hölder exponent to $H_{f}=h$, one should have

$$
S_{f}(j) \sim C 2^{-j h}
$$

for some constant $C$

which implies

$$
\log _{2} S_{f}(j) \sim-h j+C^{\prime}
$$


Let $\Psi_{j}$ denote the set of wavelet coefficients at scale $j$. The power spectrum of $f$ is defined as follows

$$
S_{f}(j)=\sqrt{\frac{1}{\# \Psi_{j}} \sum_{i, k}\left|c_{j, k}^{(i)}\right|^{2}}
$$

If $f$ is associated to a Hölder exponent to $H_{f}=h$, one should have

$$
S_{f}(j) \sim C 2^{-j h}
$$

for some constant $C$

which implies

$$
\log _{2} S_{f}(j) \sim-h j+C^{\prime}
$$

so that the Hölder exponent can be estimated using a log-log plot 
One can also determine $h$ by fitting the curve $\gamma(h, C)=C 2^{-\cdot h}$ to the function $S_{f}$ (using e.g. the Levenberg-Marquardt algorithm) 
One can also determine $h$ by fitting the curve $\gamma(h, C)=C 2^{-\cdot h}$ to the function $S_{f}$ (using e.g. the Levenberg-Marquardt algorithm)

Using the previous theorem, this method can be adapted for more general curves $\gamma(h, C)=C \omega^{(h)}\left(2^{-\cdot}\right)$ 
One can also determine $h$ by fitting the curve $\gamma(h, C)=C 2^{-\cdot h}$ to the function $S_{f}$ (using e.g. the Levenberg-Marquardt algorithm)

Using the previous theorem, this method can be adapted for more general curves $\gamma(h, C)=C \omega^{(h)}\left(2^{-\cdot}\right)$

For the Brownian motion, one is naturally led to choose

$$
\omega_{W}^{(h)}(r)=(r \log |\log r|)^{h}
$$

in order to get a sharper estimation and help to discern between two models 
For the Brownian motion $W$, the "usual" method gives $H_{W}=0.48 \pm 510^{-2}$ and the new one gives $H_{W}=0.499 \pm 310^{-2}$

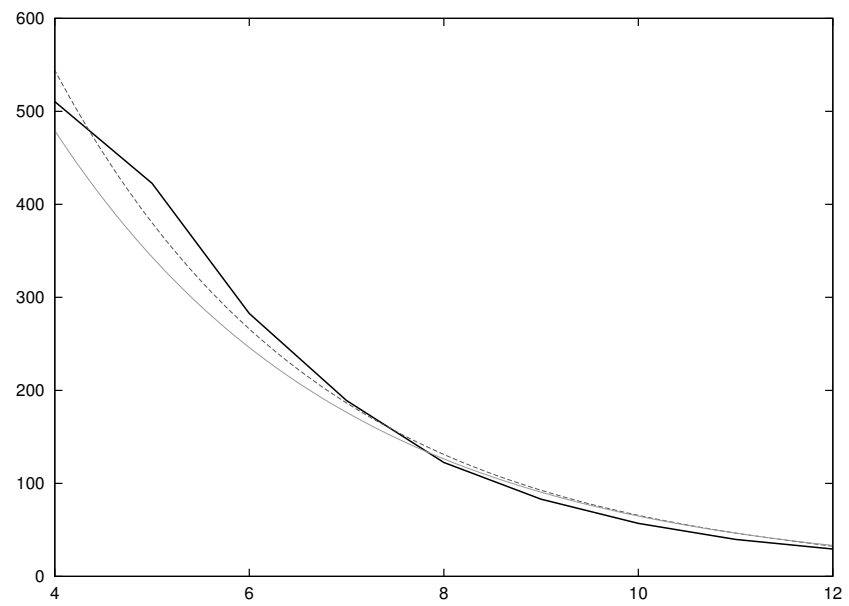

$S_{W}$ (thick black), $j \mapsto C 2^{-j h}$ (grey) and $j \mapsto \omega_{W}^{(h)}\left(2^{-j}\right)$ (dashed lines) 
If $Z_{k} \stackrel{\text { iid }}{\sim} N(0,1)$ let

$$
W_{u n i}: x \mapsto \sum_{k=0}^{\infty} \phi^{k} \cos \left(\left(\omega^{k}+Z_{k}\right) \pi\right)
$$

and

$$
W_{\text {norm }}: x \mapsto \sum_{k=0}^{\infty} Z_{k} \phi^{k} \cos \left(x \omega^{k} \pi\right)
$$

two generalizations of the Weierstraß function $(\phi \in(0,1)$ and $\phi \omega>1)$. 
If $Z_{k} \stackrel{\text { iid }}{\sim} N(0,1)$ let

$$
W_{\text {uni }}: x \mapsto \sum_{k=0}^{\infty} \phi^{k} \cos \left(\left(\omega^{k}+Z_{k}\right) \pi\right)
$$

and

$$
W_{\text {norm }}: x \mapsto \sum_{k=0}^{\infty} Z_{k} \phi^{k} \cos \left(x \omega^{k} \pi\right)
$$

two generalizations of the Weierstraß function $(\phi \in(0,1)$ and $\phi \omega>1)$.

The first process is well known to behave as the Brownian motion, while the study of the behavior of the second one has still to be carried out 


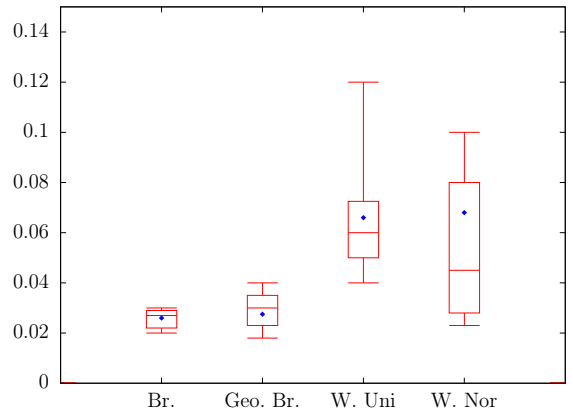

When the behavior of the process is well known (Brownian motion, geometric Brownian motion and $\left.W_{u n i}\right)$, the numerical tests confirm that the new method is able to detect a logarithmic correction 


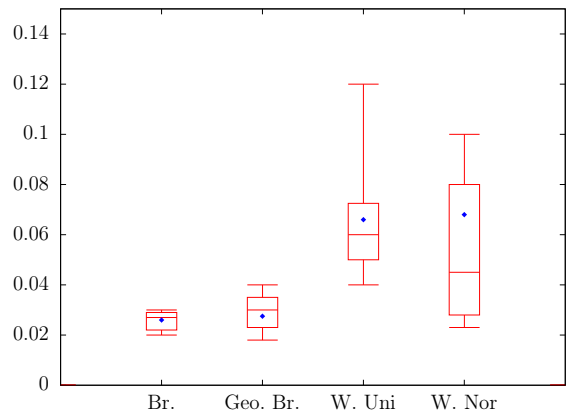

When the behavior of the process is well known (Brownian motion, geometric Brownian motion and $\left.W_{u n i}\right)$, the numerical tests confirm that the new method is able to detect a logarithmic correction

When performed on $W_{\text {norm }}$, this technique suggests that there is no logarithmic correction 